\title{
ARTí́CULO
}

\section{Listado taxonómico, aspectos ecológicos y biogeográficos de las larvas de peces del Sistema Arrecifal Veracruzano, Suroeste del Golfo de México (junio 2011-junio 2013)}

Veracruz reef system fish larvae ecological aspects, biogeography and taxonomic record, Gulf of Mexico Southwest (June 2011-June 2013)

\section{Germán A. Ayala-Rodríguez ${ }^{1 *}$, Uriel Ordóñez-López², César Meiners ${ }^{1}$ y Mark Marín-Hernández ${ }^{1}$}

\begin{abstract}
'Universidad Veracruzana, Instituto de Ciencias Marinas y Pesquerías, Calle Hidalgo 617, Col. Rio Jamapa, C.P. 94290, Boca del Rio, Veracruz, México. *man7003@gmail.com

${ }^{2}$ Centro de Investigación y de Estudios Avanzados, CINVESTAV-IPN, Antigua carretera a Progreso Km 6, Cordemex, 97310, Mérida, Yucatán, México

Abstract.- A taxonomic checklist, biogeographic data and spatio-temporal distribution for fish larvae in Veracruz Reef System, Mexico are presented. Twenty six stations were monitored monthly in the SAV with 9 samplings in total, in the period June 2011June 2013. Samples were obtained from diurnal horizontal surface tows with a conical net. Fish larvae spatio-temporal distribution and ecological index in this system are showed. Highest larvae fish abundance occurred in one month of rainy season and 2 months at the end of 'northerlies' season in nearby transects to the mouth of Jamapa River. 3941 organisms belonging to 61 taxa were obtained: 47 to level species and 14 to genus were identified. Atherinopsidae and Carangidae families showed the highest Specific Richness with 4 species each one. The highest larvae fish abundance belonged to Haemulidae family (Haemulon aurolineatum and $\mathrm{H}$. plumierii) which contributes $47.62 \%$ to total abundance. With this contribution, the geographic distribution of larvae stage of fishes Apogon aurolineatus, Cantherhines macrocerus, Cheilopogon cyanopterus, Hemiramphus brasiliensis and M embras vagrans towards central and south of Gulf of Mexico and Mexican Sea Caribbean was expanded. New records support connectivity theories and dispersal patterns postulated by several authors between reefs of western coast Gulf of Mexico and Mexican Caribbean. Rainfall and end northerlies season act as natural mechanisms that propitiate accurate conditions for maintaining high larvae abundance. It was showed a substantial number of species in this sampling, however, we suggest alternative methods for record cryptic species, due to habitat complexity in this area.
\end{abstract}

Key words: Ichthyoplankton, fish larvae, Veracruz Reef System, VRS, biogeography, connectivity

\begin{abstract}
Resumen.- Se presenta un listado taxonómico de larvas de peces del Sistema Arrecifal Veracruzano, México. Veintiseis estaciones fueron muestreadas durante 9 meses en el periodo junio 2011 a junio 2013. Las muestras se obtuvieron de arrastres horizontalessuperficiales y diurnos con una red cónica. Se muestra la distribución espacio-temporal de las larvas de peces y los índices ecológicos en este sistema. Las mayores densidades se presentaron durante un mes de la época de lluvias y 2 meses al final de temporada de nortes en transectos cercanos a la boca del Rio Jamapa. Se obtuvieron un total de 3941 organismos pertenecientes a 61 taxa, de los cuales 47 se identificaron a nivel especie y 14 a género. Las familias Atherinopsidae y Carangidae presentaron la mayor riqueza específica con 4 especies cada una. Haemulon aurolineatum y H. plumierii aportaron el 47,62\% de la abundancia total. Con esta contribución se amplía la distribución geográfica de los estadios larvarios de: Apogon aurolineatus, Cantherhines macrocerus, Cheilopogon cyanopterus, Hemiramphus brasiliensis y M embras vagrans hacia la parte central y sur del Golfo de México y el Mar Caribe Mexicano. En total, de las 47 especies identificadas, 24 representan un nuevo registro para el Sistema Arrecifal Veracruzano. Los nuevos registros encontrados en el área soportan las teorías de dispersión y conectividad entre arrecifes de la costa occidental del Golfo de México y el Caribe Mexicano propuestas por otros autores. Mecanismos naturales como el periodo de lluvias o el final de temporada de nortes propician condiciones adecuadas para mantener altas densidades larvarias en este sistema. El monitoreo mostró un número relevante de especies, sin embargo se sugieren métodos alternativos para ampliar el registro de especies crípticas, debido a la complejidad de hábitats presentes.
\end{abstract}

Palabras clave: Ictioplancton, larvas de peces, Sistema Arrecifal Veracruzano, SAV, biogeografía, conectividad 


\section{INTRODUCCIÓN}

Las zonas arrecifales son hábitats críticos relevantes, ya que proveen áreas de refugio, crianza, alimentación y reproducción para un amplio espectro de especies de invertebrados y vertebrados, incluyendo un número importante de especies de importancia comercial (Grigg et al. 1984). A pesar de la connotación ecológica y económica que poseen estas áreas, paradójicamente es donde menor conocimiento se ha generado en cuanto a taxonomía de los estadios larvarios de peces, si se comparan con las zonas lagunares costeras y marinas (Leis 1993).

En el Sistema Arrecifal Veracruzano (SAV) los estudios referentes a la composición taxonómica de las comunidades de peces se han enfocado exclusivamente a los estadios adultos de hábitos arrecifales a través de censos visuales (RangelAvalos et al. 2008, Del Moral-Flores et al. 2013) y aquellas especies de importancia comercial, monitoreadas en las localidades pesqueras aledañas al SAV (Jiménez-Badillo \& Castro-Gaspar 2007). Los estudios disponibles sobre el ictioplancton en el Golfo de México se han llevado a cabo primordialmente en cuerpos lagunares costeros y en la plataforma continental con una resolución de varias decenas de kilómetros (Flores-Coto et al. 2009, García-Hernández et al. 2009). Sin embargo, hoy en día, no se cuenta con referencias plausibles sobre la composición taxonómica del ictioplancton en el SAV.

El SAV se encuentra localizado frente al Puerto de Veracruz, Boca del Río y Antón Lizardo en el Suroeste del Golfo de México; es un área natural protegida conformada por 23 arrecifes, incluyendo 6 islas, dentro de una extensión de 65,516 $\mathrm{km}^{2}$ (DOF 2012) ${ }^{1}$. Los edificios arrecifales se encuentran divididos en 2 zonas, separadas por la pluma del río Jamapa, la zona norte con 11 arrecifes y la zona sur con 12 formaciones (Fig. 1). Por su cercanía a la costa, el SAV está sujeto a impactos derivados de actividades antropogénicas como el desfogue de las aguas de descarga de la zona urbana y conurbada del puerto de Veracruz y Boca del Rio (Ortiz-Lozano et al. 2009), además de la descarga de los ríos Papaloapan en la porción sur, Jamapa en el centro y La Antigua en la porción norte (Vargas-Hernández et al. 1993, Ortiz-Lozano et al. 2015).

En el presente estudio se describen por primera vez los componentes larvarios de los peces para el Sistema Arrecifal Veracruzano, con información sobre identidad taxonómica, abundancia, hábitos ecológicos y aspectos zoogeográficos de las especies. Todo esto derivado de muestreos diurnos superficiales, además de una comparación bibliográfica con otros trabajos faunísticos realizados en el área con registros de estadios adultos de peces, y se actualiza el registro de las distintas etapas de vida de estos organismos en el sistema.

\section{MATERIALES Y MÉTODOS}

Se recolectaron muestras de zooplancton durante 9 cruceros en el SAV de junio 2011 a junio 2013 (Fig. 1), sobre una red de 26 estaciones distribuidas en 4 transectos perpendiculares a la costa (A, B, C y D, de norte a sur). Los muestreos fueron diurnos y se realizaron mediante arrastres de zooplancton horizontales superficiales con una red cónica estándar (1,5 m largo, $0,5 \mathrm{~m}$ diámetro de boca, malla $333 \mu \mathrm{m}$ ) durante $5 \mathrm{~min}$ con un flujómetro adaptado en la boca para estimar la densidad de larvas $100 \mathrm{~m}^{-3}$ (Smith \& Richardson 1977).

Las muestras se fijaron y preservaron en formalina al $4 \%$ amortiguada con borato de sodio. Se separaron las larvas de peces y se identificaron al nivel taxonómico más bajo posible, generalmente a nivel de especie, con el apoyo de literatura especializada (Richards 2006, Fahay 2007). El hábitat de cada especie se obtuvo a partir de Richards (2006), Fahay (2007) y Froese \& Pauly (2014) y la afinidad de las especies de Froese \& Pauly (2014). Las afinidades zoogeográficas de las especies se catalogaron de acuerdo con la clasificación de regiones y provincias de Briggs (1974), el cual fue modificado por CastroAguirre et al. (1999), donde se consideró la distribución actual de las especies y su presencia en las siguientes divisiones de la región del Atlántico occidental: a) provincia Virginiana (PV): cabo Cod hasta cabo Hatteras; b) provincia Caroliniana de la costa oriental de EUA (PCO): cabo Hatteras hasta la costa sureste de Florida; c) provincia Caroliniana de la costa norte, noreste y noroeste del Golfo de México (PCM): costa suroeste de Florida hasta cabo Rojo, Tamiahua, Veracruz; d) provincia Caribeña (PC): cabo Rojo, Tamiahua, Veracruz hasta el noreste de Venezuela; e) provincia Antillana (PA): costas norte y este de la península de Yucatán, Antillas Mayores y Menores.

Se evaluó la calidad del inventario taxonómico a través del ajuste de una curva de acumulación de especies al modelo de Clench (Soberón \& Llorente 1993, Jiménez-Valverde \& Hortal 2003):

$$
\mathrm{S}_{\mathrm{n}}=\mathrm{a} * \mathrm{n} /(1+\mathrm{b} * \mathrm{n})
$$

$a=$ tasa de incremento de nuevas especies al comienzo del inventario

$b=$ parámetro de forma de la curva

$a / b=$ asíntota o número de especies a predecir por la curva

$S_{n}=$ riqueza de especies esperada 
Se estimó la riqueza de especies $\mathrm{S}$ (i) y la diversidad $\mathrm{H}^{\prime}$ (ii) (Shannon-Wiener).

i) $\mathrm{S}=$ número total de especies presentes en cada mes de muestreo para todos los transectos

ii) $H=-\Sigma p i * \ln (p i)$

$\mathrm{H}=$ índice de diversidad de Shannon-Wiener

$p i=$ proporción del número de organismos de la especie $i$ con respecto a $\mathrm{N}$ obteniendo pi de la división del número de individuos de una especie con la sumatoria del número total de individuos de todas las especies

$\ln (p i)=\log$ aritmo natural de $p i$

Para probar diferencias significativas entre las densidades promedio de larvas, valores de diversidad y riqueza especifica entre estaciones, meses y transectos de la zona, se usó la prueba no paramétrica Kruskal-Wallis $(\mathrm{H})$, después de haber probado la no normalidad de los datos con las pruebas KolmogorovSmirnov y Shapiro-Wilk (STATISTICA ${ }^{\circledR}$ v10.0 , StatSoft, OK, USA).

\section{RESUltados}

Se obtuvo un total de 3941 larvas de peces, de las cuales el 90\% (3582 larvas) fueron objeto de identificación positiva a cierto nivel taxonómico. Las restantes 359 larvas al estar en estadio de larva vitelina, fue imposible su identificación incluso a nivel de familia. El conjunto de larvas identificadas se agrupó en 61 taxa, repartidos en 1 clase, 11 órdenes, 37 familias, 53 géneros y 47 especies (Tabla 1). En total, de las 47 especies identificadas en este estudio, solo han sido reportadas 23 en el SAV, representando el 48,94\% del total de especies, y 24 especies representan un nuevo registro para el Sistema Arrecifal Veracruzano.

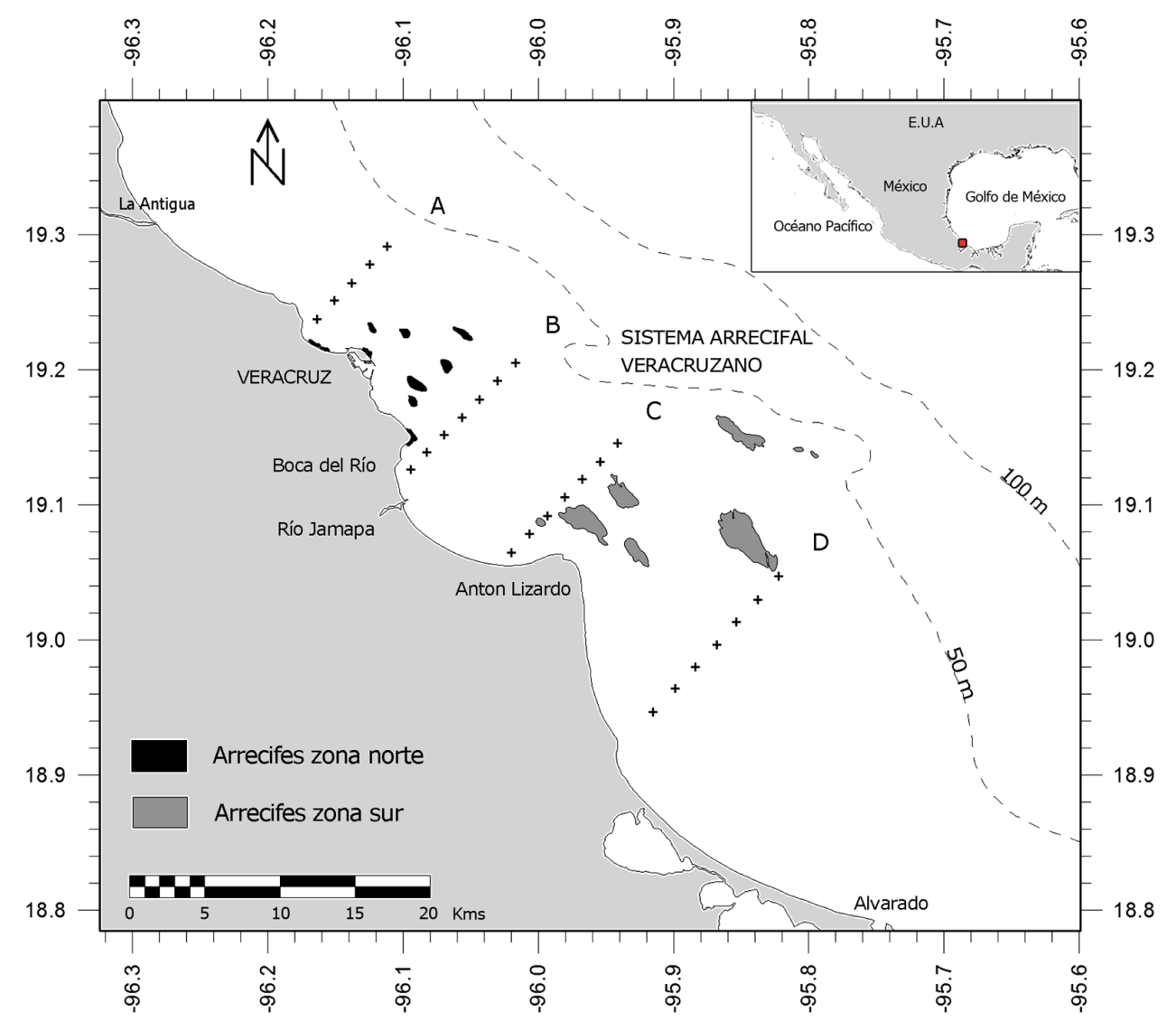

Figura 1. Localización de la red de estaciones en los 4 transectos (A, B, C, D) perpendiculares a la costa a través del Sistema Arrecifal Veracruzano. En color negro están los arrecifes de la porción norte del sistema (transectos A y B) y en gris claro los de la parte sur (transectos C y D) / Net Stations location in 4 transects off Veracruz Reef System (A, B, C, D). Black color denotes reefs group of north zone (A and B), grey light, reefs group of south zone (C and D) 
Tabla 1 Atributos ecológicos y taxonómicos de las lanvas de peces colectadas entre junio 2011 a junio 2013 en el Sistema Arrecifal

Veracruzano/ Taxonomic and ecological attributes of fish larvae collected between June 2011 and June 2013 in Veracruz Reef System

\begin{tabular}{|c|c|c|c|}
\hline Especie & \multicolumn{2}{|c|}{ Registros } & Provincias Biogeográficas \\
\hline \multirow{2}{*}{\multicolumn{4}{|c|}{ Engraulidae }} \\
\hline Anchoa hepsetus (Linnaeus, 1758) & & & PV,PCO,PCM,PA,PB,PC \\
\hline Anchoa mitchilli (Valenciennes, 1848) & 2,4 & ${ }^{* * *}$ & PV,PCO,PCM,PA,PC \\
\hline \multicolumn{4}{|l|}{ Clupeidae } \\
\hline Harengula jaguana (Poey, 1865) & $1,2,4$ & 6,10 & PV,PCO,РСM,РА,РB,РC \\
\hline Opisthonema oglinum (Lesueur, 1818) & $1,2,4$ & $* * *$ & PV,PCO,PCM,PA,PB,PC \\
\hline \multirow{2}{*}{\multicolumn{4}{|c|}{ Haemulidae }} \\
\hline & & & \\
\hline $\begin{array}{l}\text { Hacemulon anrolininatum (Cuvier, 1830) } \\
\text { Haemulon plumieri (Lacepede, 1801) }\end{array}$ & $\frac{1,3,4}{2}$ & $\begin{array}{c}6,8,9,10 \\
6,7,10\end{array}$ & $\begin{array}{l}\text { PCM,PA,PB,PC } \\
\text { PV,PCO,PCM,PA,PB,PC }\end{array}$ \\
\hline \multicolumn{4}{|l|}{ Bregmacerotidae } \\
\hline Bregmaceros cantori (Milliken $e$ Houde, 1984) & 2,5 & "*** & АТ,PCO,РCM,РA,PB,PC \\
\hline \multicolumn{4}{|l|}{ Atherinopsidae } \\
\hline Menidia beryllina (Cope, 1867) & 1,3 & **** & РСО,РСM,PC \\
\hline Menidia menidia (Linnaeus, 1766) & & ***** & $\mathrm{PV}, \mathrm{PCO}, \mathrm{PCM}, \mathrm{PA}, \mathrm{PC}$ \\
\hline $\begin{array}{l}\text { Membras martinica (Valenciennes, } 18355) \\
\text { Membras vagrans (Goode et Beanan 1879) }\end{array}$ & $1,2,3,4$ & ****** & PV,PCO,PCM,PA,PC \\
\hline \multicolumn{4}{|l|}{$\begin{array}{l}\text { Membras vagrans (Goode et Bean, 1879) } \\
\text { Apogonidae }\end{array}$} \\
\hline Apogon aurolineatus (Mowbray, 1927) & +++ & $* * * *$ & РCO,РCM,PA,PB,PC \\
\hline \multicolumn{4}{|l|}{ Hemiramphidae } \\
\hline Hemiramphus brasiliensis (Linnaeus, 1758) & +++ & $6,7,10$ & АT,PV,PCO,PCM,PA,PB,PC \\
\hline 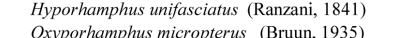 & $2,3,4$ & $*^{* * *}$ & РCO,РCM,РA,PB,PC \\
\hline \multicolumn{4}{|l|}{$\begin{array}{l}\text { Oxyporhamphus micropterus (Bruun, 1935) } \\
\text { Blenniidae }\end{array}$} \\
\hline $\begin{array}{l}\text { Blennudae } \\
\text { Hypsoblennius hentz (Lesueur, 1825) }\end{array}$ & & $* * *$ & PV,PCO,PCM,PA,PC \\
\hline Lupinoblennius nicholsi (Tavolga, 1954) & $1,2,4$ & 10 & PCO,PCM,PA,PC \\
\hline \multicolumn{4}{|l|}{ Kyphosidae } \\
\hline $\begin{array}{l}\text { Kyphosus sectatrix (Linnaeus, 1758) } \\
\text { Snaridae }\end{array}$ & 1 & $6,7,9,10$ & AT,PV,PCO,PCM,PA,PC \\
\hline $\begin{array}{l}\text { Sparidae } \\
\text { Lagodon rhomboides (Linnaeus, 1766) }\end{array}$ & 2.3 .4 .5 & 6,10 & PV.PCO,PCM.PA.PC \\
\hline Paralichthyidae & & & \\
\hline Syacium gunteri (Ginsburg, 1933) & 2,5 & 10 & $\mathrm{PCO}, \mathrm{PCM}, \mathrm{PA}, \mathrm{PC}$ \\
\hline \multicolumn{4}{|l|}{ Sphyraenidae } \\
\hline \multicolumn{4}{|l|}{$\begin{array}{l}\text { Sphyraena barracuda (Edwards, 1771) } \\
\text { Pomacentridae }\end{array}$} \\
\hline 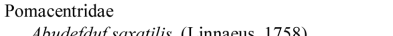 & & & \\
\hline $\begin{array}{l}\text { Abudefduf saxatilis (Linnaeus, 1758) } \\
\text { Stegastes sp. (Jenyns, 1840) }\end{array}$ & $1,2,3$ & $6,8,9,10$ & АT,PV,PCO,PCM,PA,PB,PC \\
\hline \multicolumn{4}{|l|}{$\begin{array}{l}\text { Jisegastessps., (enyns, } 1840) \\
\text { Tetragonuridae }\end{array}$} \\
\hline \multirow{2}{*}{\multicolumn{4}{|c|}{ Exocoetidae }} \\
\hline & & & \\
\hline Cheilopogon cyanopterus (Valenciennes, 1847) & +++ & $* * *$ & $\mathrm{CT}$ \\
\hline \multirow{2}{*}{\multicolumn{4}{|c|}{ Carangidae }} \\
\hline & & & \\
\hline $\begin{array}{l}\text { Decapterus sunctatus (Cuvier, } 1829 \text { ) } \\
\text { Oligoplites saurus (Bloch } e \text { Schneider, 1801) }\end{array}$ & 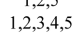 & 10 & $\mathrm{AA}, \mathrm{PV}, \mathrm{PCO}, \mathrm{PCM}, \mathrm{PA}, \mathrm{PB}, \mathrm{PC}$ \\
\hline Selar crumenophthalmus (Bloch, 1793) & $1,2,5$ & 6,10 & $\begin{array}{ll}\text { CT } \\
\end{array}$ \\
\hline \multirow{2}{*}{\multicolumn{4}{|c|}{ Mugilidae }} \\
\hline & & & \\
\hline Mugil cephalus ( Linnaeus, 1758) & $1,2,3,4,5$ & $7,9,10$ & CT \\
\hline Mugili curema (Valenciennes, 1836) & $1,2,3,4$ & 6,10 & $\mathrm{AA}, \mathrm{AT}$ \\
\hline
\end{tabular}

\begin{tabular}{|c|c|c|c|}
\hline \multirow{2}{*}{ Especie } & \multicolumn{2}{|c|}{ Registros } & \multirow{2}{*}{ Provincias Biogeográficas } \\
\hline & Larvas & Adultos & \\
\hline $\begin{array}{l}\text { Gerreidae } \\
\text { Eucinostomus argenteus (Baird et Girard, 1855) } \\
\text { Eucicostomus sp. (Baird, 1857) }\end{array}$ & $1,3,4$ & 7,10 & $\mathrm{PCM}, \mathrm{PA}, \mathrm{PB}, \mathrm{PC}$ \\
\hline Monacanthidae & & & \\
\hline $\begin{array}{l}\text { Cantherhines macrocerus (Hollard, 1853) } \\
\text { Stephanolepis setfifer (Bennett. 1831) }\end{array}$ & +++ & 10 & $\begin{array}{c}\text { PCM,PA,PB,PC } \\
\text { PCO PCM PA }\end{array}$ \\
\hline $\begin{array}{l}\text { Stephanolepis setifer (Bennett, 1831) } \\
\text { Synodontidae }\end{array}$ & & & AA,PCO,PCM,PA,PB,PC \\
\hline $\begin{array}{l}\text { Synodus foetens (Linnaeus, 1766) } \\
\text { Scionido }\end{array}$ & $2,3,5$ & 6,10 & PCM,PA,PB,PC \\
\hline $\begin{array}{l}\text { Sciaenidae } \\
\text { Cynoscion nebulosus (Cuvier, 1830) } \\
\text { Cynoscion regalis (Bloch et Schneider, 1801) }\end{array}$ & $\frac{2,3,4}{3}$ & $\begin{array}{l}* * * \\
* * *\end{array}$ & $\begin{array}{l}\text { PV,PCO,PCM,PA,PC } \\
\text { PVVPCOPCM,PA }\end{array}$ \\
\hline Bothidae & & & \\
\hline Bothus ocellatus (Agassiz, 1831) & $1,2,5$ & 6,10 & $\mathrm{PV}, \mathrm{PCO}, \mathrm{PCM}, \mathrm{PA}, \mathrm{PB}, \mathrm{PC}$ \\
\hline $\begin{array}{l}\text { Coryphaenidae } \\
\text { Coryphaeena equiselis (Linnaeus, 1758) } \\
\text { Corrvphaena sp. Linnaeus, 1758) }\end{array}$ & 2 & *** & 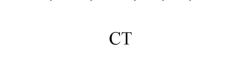 \\
\hline $\begin{array}{l}\text { Coryphaena sp. ( Linnaeus, 1758) } \\
\text { Dactylopteridae }\end{array}$ & & & \\
\hline $\begin{array}{l}\text { Dactlolopterus volitans (Linnaeus, 1758) } \\
\text { Belonidae }\end{array}$ & 2 & ${ }^{* * *}$ & AT,PV,PCO,PCM,PA,PB,PC \\
\hline $\begin{array}{l}\text { Belonidae } \\
\text { Strongylura marina (Walbaum, 1792) }\end{array}$ & $1,2,3,4$ & $* * *$ & PV,PCO,PCM,PA,PB,PC \\
\hline Tylosurus crocodilus (Péron et Lesueur, 1821) & 1,3 & $6,9,10$ & \\
\hline $\begin{array}{l}\text { Achiridae } \\
\text { Achirus lineatus (Linnaeus, 1758) }\end{array}$ & 1245 & $* * * *$ & РСОРСМРА РВ РС \\
\hline 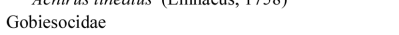 & & & C \\
\hline $\begin{array}{l}\text { Gobiesox strumosus (Cope, 1870) } \\
\text { Antennarida. }\end{array}$ & $1,2,3,4$ & 10 & $\mathrm{PV}, \mathrm{PCO}, \mathrm{PCM}, \mathrm{PA}, \mathrm{PB}, \mathrm{PC}$ \\
\hline $\begin{array}{l}\text { Antennariidae } \\
\text { Histrio histrio (Linnaeus, 1758) }\end{array}$ & 2 & 6,10 & CT \\
\hline Ophichthidae & & & \\
\hline $\begin{array}{l}\text { Ophichthus cruentifer (Goode et Bean, 1896) } \\
\text { Gobiidae }\end{array}$ & 2,5 & $* * *$ & $\mathrm{PV}, \mathrm{PCO}, \mathrm{PCM}, \mathrm{PA}, \mathrm{PC}$ \\
\hline $\begin{array}{l}\text { Gobididae } \\
\text { Bathygobius sp. (Bleeker, 1878) }\end{array}$ & & & \\
\hline $\begin{array}{l}\text { Gobionellus sp. (Girard, 1858) } \\
\text { Gobiosoma sp. (Lacepède, 1800) }\end{array}$ & & & \\
\hline Serranidae & & & \\
\hline Diplectrum sp. (Holbrook, 1855) & & & \\
\hline Serranus sp. (Cuvier, 1817) & & & \\
\hline $\begin{array}{l}\text { Tripterygiidae } \\
F \text { ropoctos }\end{array}$ & & & \\
\hline $\begin{array}{l}\text { Enneanectes sp. (Jordan et Gilbert, 1895) } \\
\text { Scorpacenidac }\end{array}$ & & & \\
\hline $\begin{array}{l}\text { Scorpaanidac } \\
\text { Scorpaena sp. (Linnaeus, 1758) }\end{array}$ & & & \\
\hline $\begin{array}{l}\text { Scorpdena sp. (Linnaeus, 1/38) } \\
\text { Labrisomidae }\end{array}$ & & & \\
\hline Labrisomus sp. (Swainson, 1839) & & & \\
\hline Microdesmidae & & & \\
\hline $\begin{array}{l}\text { Microdesmus sp. (Günther, 1864) } \\
\text { Onbididese }\end{array}$ & & & \\
\hline $\begin{array}{l}\text { Ophidiidae } \\
\text { Ophidium sp.(Linnaeus, 1766) }\end{array}$ & & & \\
\hline $\begin{array}{l}\text { Oppidium sp. (Linnaeus, 1766) } \\
\text { Labridae }\end{array}$ & & & \\
\hline Thalassoma sp. (Swainson, 1839) & & & \\
\hline
\end{tabular}

Clave de las afinidades zoogeográficas: PV (provincia Virginiana); PCO (provincia Caroliniana de la costa oriental); PCM (provincia Caroliniana de la costa norte, noreste y noroeste del Golfo de México); PC (provincia Caribeña); PA (provincia Antillana); PB (provincia Brasileña); CT (Circumtropical); AT (Anfiatlántica); AA (Anfiamericana). Registro de larvas de peces reportadas en áreas del Golfo de México: 1 = Álvarez-Cadena et al. (2007), Quintana Roo; 2 = Flores-Coto et al. (2009), sur del Golfo de México (Campeche); 3 = García-Hernández et al. (2009), Norte de la Península de Yucatán; 4 = Sanvicente-Añorve et al. (2011), 7 lagunas costeras del Golfo de México y Caribe Mexicano; 5 = Espinosa-Fuentes et al. (2013), Plataforma continental de Tabasco y Campeche. Registros de adultos reportados en el SAV: 6 = Vargas-Hernández et al. (2002); 7 = Jiménez-Badillo y Castro-Gaspar (2007); $8=$ Martínez-Hernández y Camacho-Olivares (2007); 9 = Rangel-Avalos et al. (2008); 10 = Del Moral-Flores et al. (2013). Los símbolos (+++) muestran las especies de peces en estadio larvario sin registro en el centro y sur del Golfo de México y el Mar Caribe Mexicano. Los símbolos $(* * *)$ muestran las especies reportadas en este estudio, que no han sido registradas previamente en su estadio adulto en el SAV. A partir de la familia Gobiidae hasta Labridae no se hicieron comparaciones con registros previos en el área, debido a que en estas familias los organismos solo fueron identificados a nivel de género 
Las familias que presentaron el más alto número de especies fueron: Atherinopsidae y Carangidae con 4 especies cada una. Las larvas de peces con una mayor abundancia en el SAV pertenecieron a la familia Haemulidae con 2 especies, y representaron el $47,62 \%$ del total de los organismos muestreados. De éstos, Haemulon aurolineatum contribuyó con un $28,97 \%$ y H. plumierii con $18,64 \%$. Las familias que contribuyeron con porcentajes importantes a la abundancia, en orden descendente fueron: Clupeidae (17,3\%), Atherinopsidae $(9,2 \%)$, Engraulidae $(6,6 \%)$, Blenniidae $(5 \%)$ y Hemiramphidae $(3,4 \%)$.

El elenco taxonómico descrito en este trabajo correspondió al $86 \%$ de los taxa de larvas de peces de hábitos diurnos y superficiales en el SAV de acuerdo al modelo teórico de Clench, a través del cual se estima en 70 los taxa probables de encontrar. La curva de acumulación de especies (Fig. 2) fue significativa $(\mathrm{b}<0,1)$ y mostró un buen ajuste a los datos observados $\left(R^{2}=0,988\right)$.

El hábitat de las especies del SAV en este estudio mostró que $63 \%$ corresponden a especies de hábitos arrecifales, seguido por las de hábitos costero-pelágicos $(15,2 \%)$, epipelágicos $(15,2 \%)$ y por último las demersales que contribuyeron con el 6,5\% (Fig. 3). Del total de especies identificadas en el estudio (47), el 69,5\% correspondieron a las subtropicales, seguidas por el $26 \%$ de afinidad tropical y solo una pequeña parte $(4,3 \%)$ fueron de afinidad templada (Fig. 4).

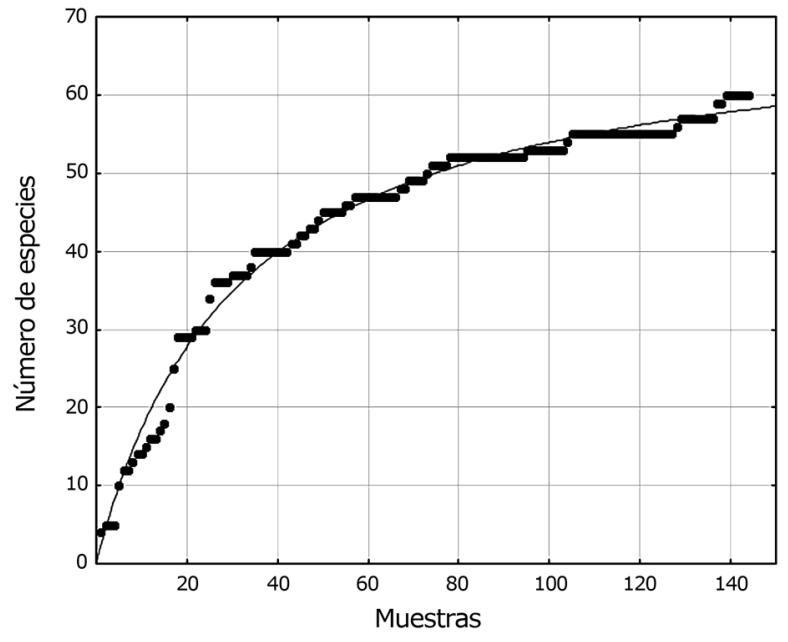

Figura 2. Curva de acumulación de especies de larvas de peces del Sistema Arrecifal Veracruzano para todo el periodo muestreado. $\mathbf{S}_{\mathrm{obs}}=$ $60, R^{2}=0,98859, a / b=70,562$, pendiente $(b)=0,03251$, porcentaje de varianza explicada $\mathbf{9} \mathbf{9 8 , 8 5 9 2 7} \%$ / Species accumulation curve of fish larvae of Veracruz Reef System in whole study sampling period. $\mathrm{S}_{\mathrm{obs}}=$ $60, R^{2}=0.98859, a / b=70.562$, slope $(b)=0.03251$, explained percent variance $=98.85927 \%$

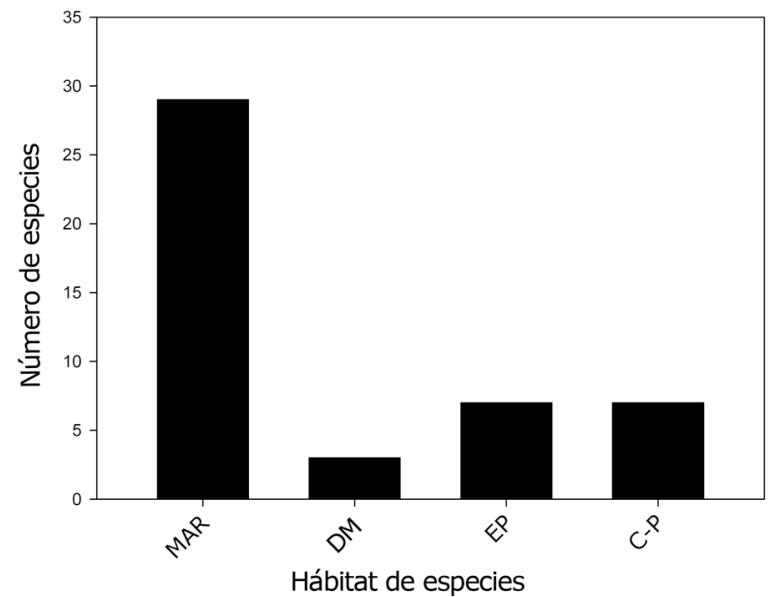

Figura 3. Hábitat de las especies (por número) de larvas de peces encontradasen el SAV durante el periodo de muestreo. $M A R=M$ arino Asociado a Arrecife, $\mathrm{C}-\mathrm{P}=$ Costero-Pelágico, $\mathrm{EP}=$ Epipelágico, $\mathrm{DM}=$ Demersal / Species habitat (by number) of fish larvae of Veracruz Reef System in whole study sampling period. MAR= Marine Reef Associated, $\mathrm{C}-\mathrm{P}=$ Coastal-Pelagic, $\mathrm{EP}=$ Epipelagic, $\mathrm{DM}=$ Demersal

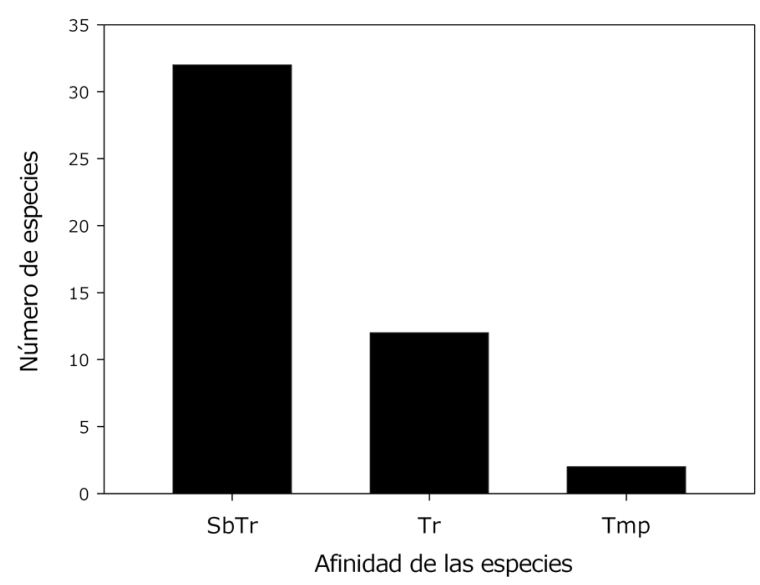

Figura 4. Afinidad de las especies de larvas de peces encontradas en el SAV durante el periodo de muestreo. SbTr=subtropical; $\mathrm{Tr}=$ tropical; Tmp = templado / Affinity of fish larvae species of Veracruz Reef System in whole study sampling period. $\mathrm{SbTr}=$ subtropical; $\mathrm{Tr}=$ tropical; $\mathrm{Tmp}=$ temperate 
La densidad de larvas difirió significativamente entre meses $(\mathrm{H}=71,34 ; \mathrm{g} .1 .=8 ; P<0,00001)$, no así entre estaciones $(\mathrm{H}=$ $2,77$; g.l. $=5 ; P=0,73)$ y transectos $(\mathrm{H}=5,408 ;$ g.l. $=3 ; P=$ $0,14)$. Los promedios de las larvas de peces en los transectos fueron más altos durante agosto 2011 y marzo 2012 y 2013 con 233, 175 y 149 larvas $100 \mathrm{~m}^{-3}$ respectivamente, donde los valores de más alta densidad se registraron en el transecto B (Fig. 5). La mayor densidad promedio de larvas por transecto de todo el periodo de estudio se presentó en agosto de 2011, y la menor en enero de 2013. Los meses restantes presentaron valores promedio menores a 60 larvas $100 \mathrm{~m}^{-3}$ (Fig. 5). En general, los transectos más cercanos a la boca del Rio Jamapa presentaron los más altos valores promedio de densidad. La riqueza promedio de especies (S) en el SAV osciló entre 1 y 10 especies por transecto. En marzo 2013 se alcanzó el máximo valor (10) seguido de marzo 2012 y junio 2013 con promedios de 7 especies cada uno. En general, los más altos valores de riqueza se obtuvieron en los transectos cercanos a la boca del río Jamapa (Fig. 6), coincidentes con valores altos de diversidad y abundancia de larvas en el sistema. La riqueza de especies difirió significativamente entre meses $(\mathrm{H}=52,95 ; \mathrm{g} .1=8 ; P<$ $0,00001)$, pero no entre estaciones $(\mathrm{H}=4,45 ; \mathrm{g} .1 .=5 ; P=0,48)$ y transectos $(\mathrm{H}=2,93 ; \mathrm{g} .1 .=3 ; P=0,401)$.

En general la diversidad $\left(\mathrm{H}^{\top}\right)$ mensual fue baja, con valores que oscilaron entre 0,2 y 1,65 nats ind ${ }^{-1}$ (Fig. 7). La máxima diversidad ocurrió en marzo de 2013 (1,4 nats ind $\left.{ }^{-1}\right)$ y coincidió con una de las más altas densidades registradas y una alta riqueza de especies $(9,6)$. La diversidad difirió significativamente entre meses $(\mathrm{H}=41,75 ; \mathrm{g} .1 .=8 ; P<0,00001)$, pero no entre estaciones $(\mathrm{H}=5,33$; g.l. $=5 ; P=0,37)$ y transectos $(\mathrm{H}=4,37$; g.l. $=3 ; P=0,22)$.

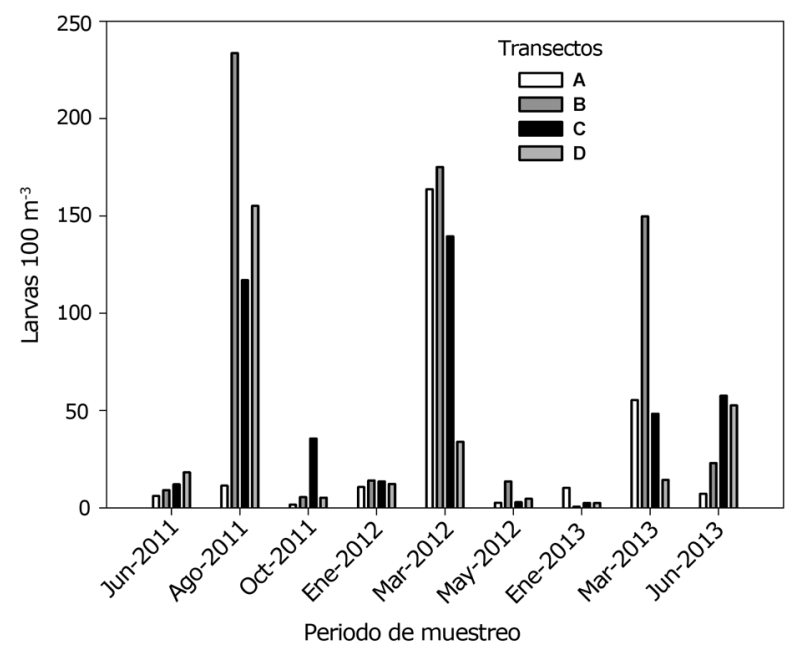

Figura 5. Densidad promedio por transecto de larvas normalizada a larvas $100 \mathrm{~m}^{-3}$ en el Sistema Arrecifal Veracruzano en todo el periodo de estudio / Fish larvae mean density by transect normalized to larvae $100 \mathrm{~m}^{-3}$ in Veracruz Reef System in whole study period
Las afinidades zoogeográficas de las especies (Tabla 1) fueron la provincia Caroliniana de la costa norte, noreste y noroeste del Golfo de México (80,85\%), seguido por la provincia Caribeña $(78,72 \%$ ) y la Antillana $(76,59 \%)$.

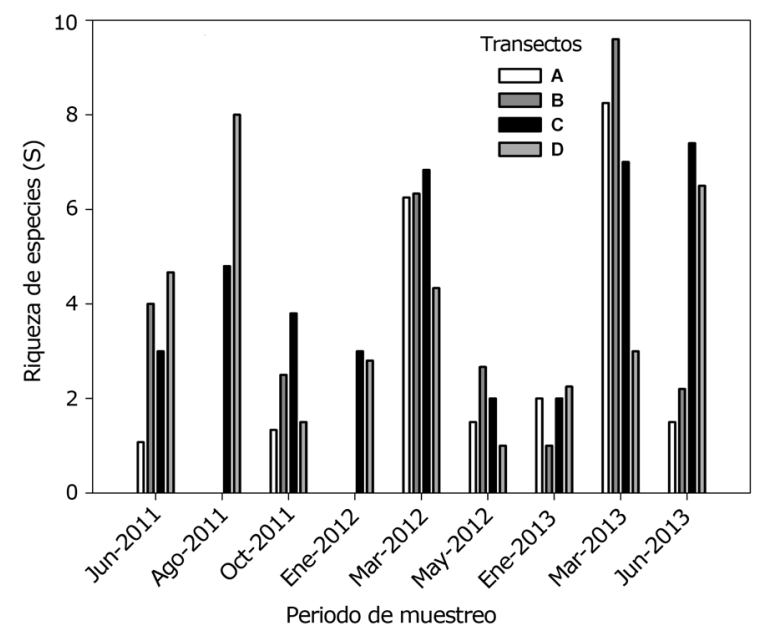

Figura 6. Riqueza promedio por transecto de especies de larvas de peces (S) en el Sistema Arrecifal Veracruzano durante todo el periodo de estudio / Mean transect species richness of fish larvae (S) of Veracruz Reef System in whole study sampling period

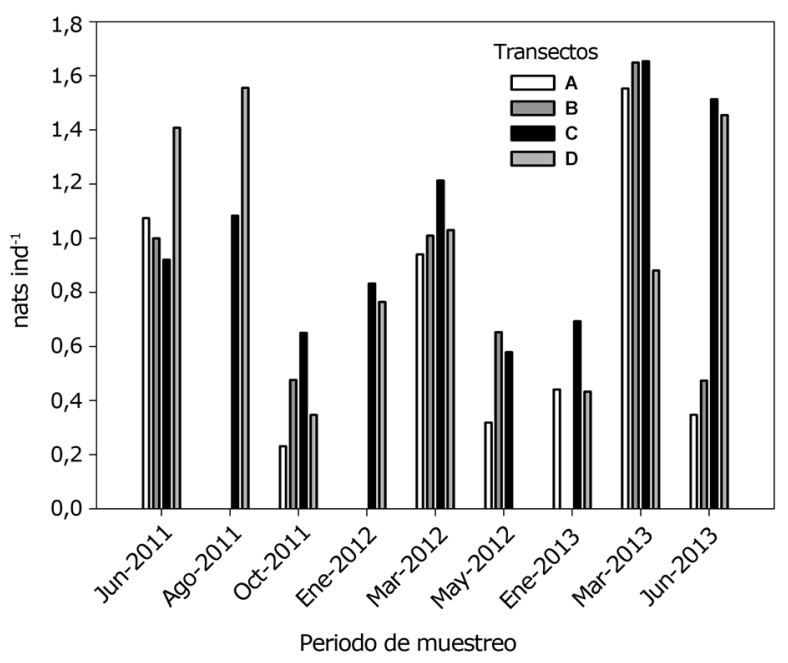

Figura 7. Promedios mensuales por transecto del Índice de diversidad de Shannon-Wiener en el Sistema Arrecifal Veracruzano durante todo el periodo de estudio / Shannon-Wiener diversity index monthly transect average of Veracruz Reef System in whole study sampling period 


\section{Discusión}

El estudio sistemático y prolongado de los estadios tempranos de peces en hábitats críticos como los sistemas arrecifales, es una cuestión relevante, ya que estos sistemas constituyen reservorios complejos de especies de peces de alto interés comercial y son áreas de crecimiento de especies clave, ecológicamente hablando (Leis 2006). En consecuencia, estas zonas arrecifales son de gran importancia para las poblaciones humanas que se dedican a la actividad pesquera en las áreas adyacentes, como sucede en el SAV, donde se explotan comercialmente más de 100 especies de peces aproximadamente y la biomasa de pesca promedio diaria oscila alrededor de 2 ton (Jiménez-Badillo \& Castro-Gaspar 2007, Dávila-Camacho et al. 2015).

Una de las etapas más críticas para la sobrevivencia de los peces es su fase larval pelágica y se ha sugerido que el tamaño de la población adulta está determinado en gran medida por la sobrevivencia de la fase pelágica. Para el caso de los peces arrecifales, durante esta etapa están expuestos a tasas de depredación elevadas, lo cual los obliga a desarrollar estrategias para maximizar la sobrevivencia de los reclutas a las fases juveniles de asentamiento (Doherty et al. 2004).

En general, hubo una correspondencia por debajo del $50 \%$ entre los estadios larvarios y los adultos registrados en el SAV, lo que puede indicar que esta área funciona como refugio y crianza de especies que no son residentes permanentes del sistema arrecifal, que se reproducen cerca de la costa, de los arrecifes o en áreas costeras aledañas, incluyendo los estuarios, y que muy probablemente tienen huevos pelágicos que son advectados desde sus áreas de origen por corrientes y posteriormente retenidos por giros o mecanismos de circulación local inducida por las formaciones arrecifales. Villegas-Sánchez et al. (2014) sugieren incluso que la presencia de estos giros producidos por la rectificación de flujo por forzamiento batimétrico inhiben el intercambio de agua y poblaciones planctónicas entre los arrecifes (Salas-Monreal et al. 2009), y pueden restringir el flujo genético entre poblaciones (e.g., Stegastes partitus) entre los arrecifes de la zona sur y norte del SAV. No obstante estas restricciones locales, previsiblemente pudieran aplicar solo para peces pequeños y eminentemente crípticos, Villegas-Sánchez et al. (2014) al mismo tiempo demostraron que existe conectividad regional, entre el Caribe Mexicano(CM) y el SAV, expresada en similitudes genéticas (microsatélites) entre juveniles de $S$. partitus entre arrecifes de la zona sur del SAV y un arrecife de la zona norte del CM.

El mecanismo probable que explica el patrón de dispersión de estas larvas de peces es el giro de Campeche, el cual impulsa la advección de organismos planctónicos desde la plataforma de Yucatán, cuando la corriente costera fluye hacia el norte permitiendo el transporte de organismos planctónicos. Este patrón de corrientes puede transportar larvas por más de 1000 km desde el CM al SAV (Salas-Pérez et al. 2012). Los peces de sistemas arrecifales poseen una fase larval pelágica de 28 a 50 días, que les permiten viajar grandes distancias desde su sitio natal a los lugares de asentamiento (Sale 2004, Bay et al. 2006). Las especies A. aurolineatus (Familia Apogonidae), C. cyanopterus (Familia Exocoetidae) y M. vagrans (Familia Atherinopsidae) no habían sido registradas previamente en la parte central y sur del Golfo de México en sus estadios larvarios, ni en fase adulta (en el SAV), por lo que probablemente ocurran procesos de dispersión que pudieran permitir el flujo de estas poblaciones desde el CM hasta el Golfo de México suroccidental.

Los estudios ictiológicos realizados en el SAV y sus áreas de influencia han hechoénfasis en el estadio adulto de los peces, con trabajos de índole ecológico y pesquero principalmente (Vargas-Hernández et al. 2002, Jiménez-Badillo \& CastroGaspar 2007, Martínez-Hernández \& Camacho-Olivares 2007, Rangel-Avalos et al. 2008, Del Moral-Flores et al. 2013, Dávila-Camacho et al. 2015), por lo que los organismos identificados en este estudio, no han sido objeto de registro anteriormente en su etapa larvaria en el SAV, en consecuencia no existen referencias directas al respecto para este sistema. El presente estudio presenta una similitud entre larvas y adultos del 5,94\% (23 especies) con el estudio más completo de este sistema hecho por Del Moral et al. (2013), quienes presentan un listado de 387 especies en un periodo de 5 años de observaciones, complementado con una revisión bibliográfica sobre peces en estadio adulto del SAV. En este trabajo, se añaden al listado taxonómico de la zona, 24 especies de peces en fase ictioplanctónica y dado que no han sido registradas anteriormente en su estadio adulto en el SAV, éstas representan nuevos registros para la zona.

De acuerdo a la curva de acumulación de especies, se establece que este muestreo de larvas de peces con hábitos diurnos superficiales fue significativo y representativo, ya que se alcanzó el 86\% (60 taxa) del elenco probable. Incluso, con el 55\% (80 muestras) del esfuerzo de muestreo, se lograría el $75 \%$ del elenco probable de larvas de peces. Bajoeste contexto, para aproximarse al 95\% ( 67 taxa) del elenco probable, se requeriría realizar 434 arrastres adicionales, lo que representa analizar un $290 \%$ más de muestras, para incrementar tan solo un $7 \%$ del elenco de larvas de peces de hábitos diurnos superficiales. La disparidad entre el número de especies presentado en este trabajo y el estudio de Del Moral-Flores et al. (2013), se debe principalmente a los métodos de colecta 
utilizados y al conjunto de publicaciones revisadas, ya que a pesar de que el modelo de Clench indica que el muestreo es representativo, está claro que están subrepresentadas las larvas de hábitos nocturnos, crípticos y/o asociados a cualquier otra condición diferente al objeto del presente estudio, por tanto la diversificación metodológica de muestreo en oportunidades subsecuentes permitirá registrar un mayor número de especies para la zona, debido a la complejidad de los hábitats arrecifales.

El estudio de peces juveniles y adultos mediante censos visuales en las inmediaciones de los arrecifes, confiere ventaja comparativa en cuanto al universo probable de especies, ya que es posible identificar y contabilizar peces con hábitos muy diferentes entre sí, ya que el SAV es un sistema heterogéneo y complejo, formado por especies crípticas y de hábitos arrecifales mayormente (en este estudio constituyen el 63\% de las especies totales).

Una de las principales limitantes de los muestreos en zonas arrecifales es la complejidad en los ciclos de vida y las formas de reproducción de los peces arrecifales (Leis 2006). Se han planteado diversos tipos de métodos de captura complementarios para estudiar las larvas de peces en este tipo de hábitats, como trampas de luz (Ordoñez-López et al. 2013), redes Renfro (García-Hernández et al. 2009), bombas de plancton (Brander \& Thompson 1989) y redes conducidas por buzos (Brogan 1994). La utilización de una red con una luz de malla mayor como $505 \mu \mathrm{m}$ pudiera ser de gran ayuda para capturar organismos de mayor tamaño, ya que en el presente estudio, una gran parte de los organismos colectados (10\% del total) estaban en etapa de preflexión y sus caracteres taxonómicos no fueron fácilmente visibles, razón por la cual no fue posible identificarlos a nivel especie (14 taxa). Uno de los métodos comúnmente utilizados son las trampas de luz, debido a que se pueden muestrear grandes volúmenes de agua y larvas de mayor tamaño, además de que se pueden obtener larvas de diferentes estratos de profundidad, a diferencia de un solo plano horizontal (Leis \& McCormick 2002).

Los valores promedio más altos de densidad de larvas de peces registrados en este estudio, coincidieron principalmente con un mes del periodo de temporada de lluvias (agosto 2011) y 2 meses (marzo 2012 y marzo 2013) correspondientes al final de temporada de nortes (Salas-Pérez \& Granados-Barba 2008). Estas densidades máximas de larvas de peces durante el periodo de lluvias en el SAV, deben estar relacionadas con los picos de abundancia zooplanctónica que han sido reportadas en este sistema (Okolodkov et al. 2011), ya que constituyen el soporte principal de una mayor disponibilidad de alimento para las larvas. Este patrón coincide con otras áreas del Golfo de México, como la zona costera de Campeche donde se han registrado las más altas biomasas zooplanctónicas y de ictioplancton durante el periodo de lluvias (Sanvicente-Añorve 1990, Sánchez-Velasco et al. 1996), y además se han registrado grandes concentraciones de ortofosfatos y silicatos que incrementan la productividad primaria, que es la principal fuente de soporte para el mantenimiento del zooplancton (PootDelgado et al. 2015).

Se ha documentado que columnas de agua bien mezcladas y con mucha turbulencia como las que ocurren en la temporada de nortes, imposibilitan a las larvas de peces en sus capacidades natatorias (Bakun 1996, Dower et al. 1997), mermando la habilidad de las mismas para capturar las partículas de alimento, lo cual resulta en un proceso de inanición y deceso probable (Cushing 1989). El patrón encontrado en este estudio al final de temporada de nortes (marzo 2012 y marzo 2013), es consistente con este proceso, ya que durante los meses donde son más fuertes los vientos del norte se observa una disminución en el número de larvas en la columna de agua y el decremento de la intensidad de los vientos a finales de la temporada de Nortes (Salas-Pérez \& Granados-Barba 2008), favorece la estratificación de la columna de agua y con ello, las larvas encuentran un ambiente más propicio para alimentarse, lo cual resulta en un incremento notable en el número de larvas en el SAV. Por otra parte, los tiempos de desove de los peces deben mostrar sincronía con procesos de dispersión favorables (Bakun 1996) y también las larvas deben asegurar su dispersión, retención y retorno a las áreas de alimentación y crecimiento, a través de estrategias como la predictibilidad y comportamiento, así como también con la ayuda de estructuras oceanográficas recurrentes (Urho 1999).

La distribución heterogénea de las larvas de peces en el SAV puede estar explicada por la compleja circulación local, la influencia variable de la descarga del río Jamapa, la variabilidad batimétrica determinada por los macizos arrecifales y la presencia de giros ciclónicos y anticiclónicos, que modifican los campos de circulación costera en esta zona (Salas-Pérez \& Granados-Barba 2008). En conjunto, estos elementos pueden jugar un papel preponderante en la distribución a escala fina de las larvas de peces, ya que los procesos de retención-dispersión son determinantes durante la fase larval pelágica para alcanzar la edad adulta (Leis 2006).

La provincia predominante fue la Caroliniana de la costa norte, noreste y noroeste del Golfo de México con 80,85\%. En segundo lugar estuvola provincia Caribeña con un porcentaje en similitud de 78,72\%, los reportes sitúan a esta provincia con el más alto grado de similitud con la fauna de distintos arrecifes del SAV, y se sugiere que estos son parecidos en composición a los arrecifes caribeños (Reséndez-Medina 1971, VillalobosFigueroa 1971, Del Moral-Flores et al. 2013). 
El listado objeto de este trabajo, representa la primera contribución pormenorizada acerca de la ocurrencia de larvas de peces de hábitos diurnos y superficiales del Sistema Arrecifal Veracruzano, así como el incremento del rango de distribución geográfico de 5 especies para el Golfo de México, Mar Caribe Mexicano y el mismo SAV. Además, proporciona elementos básicos acerca de la temporalidad de la abundancia de larvas de peces y algunos mecanismos probables que las gobiernan. A partir de esta primera contribución es indispensable profundizar en el estudio de la dinámica de ocurrencia y distribución de grupos específicos y de los procesos que determinan la variabilidad espacial y temporal de las larvas dentro del sistema arrecifal.

\section{Agradecimientos}

Se agradece el apoyo del proyecto PROMEP/103.5/10/5006 'Parámetros oceanográficos y su influencia sobre el transporte y abundancia del ictioplancton dentro del PNSAV' y al proyecto CONACyT 158152 'Estructura y variabilidad de temperatura, salinidad y corrientes dentro del PNSAV' bajo el cual fueron obtenidas las muestras biológicas. También se agradece a los fondos extraordinarios CONACyT y PIFI por el apoyo para la realización de una estancia en CINVESTAV-IPN sobre taxonomía del ictioplancton y la adquisición de material de apoyo para el desarrollo de esta investigación. Los autores agradecen los comentarios y sugerencias de los revisores anónimos que ayudaron a mejorar este manuscrito.

\section{LITERATURA CITADA}

Álvarez-Cadena JN, U Ordóñez-López, AR AlmaralMendivil, M Ornelas-Roa \& A Uicab-Sabido. 2007. Larvas de peces del litoral del norte de Quintana Roo, Mar Caribe de México. Hidrobiológica 17(2): 139-150.

Bakun A. 1996. Patterns in the ocean: ocean processes and marine population dynamics, 323 pp. California Sea Grant System, NOAA and Centro de Investigaciones Biológicas del Noroeste, La Jolla.

Bay LK, RH Crozier \& MJ Caley. 2006. The relationship between population genetic structure and pelagic larval duration in coral reef fishes on the Great Barrier Reef. Marine Biology 149: 1247-1256.

Brander K \& AB Thompson. 1989. Diel differences in avoidance of three vertical profile sampling gears by herring larvae. Journal of Plankton Research 4: 775-784.

Briggs JC. 1974. Marine zoogeography, 475 pp. McGraw-Hill, New York.

Brogan MW. 1994. Two methods of sampling fish larvae over reefs: a comparison from the Gulf of California. Marine Biology 118: 33-44.

Castro-Aguirre JL, HS Espinosa-Pérez \& JJ SchmitterSoto. 1999. Ictiofauna estuarino-lagunar y vicaria de México, 711 pp. Limusa / Instituto Politécnico Nacional, México.
Cushing D. 1989. A difference in structure between ecosystems in strongly stratified waters and in those that are only weakly stratified. Journal of Plankton Research 11: 1-13.

Dávila-Camacho CA, P Arceo, H Pérez-España \& J BelloPineda. 2015. Las pesquerías del Sistema Arrecifal Veracruzano SAV bajo un enfoque ecosistémico. E-Bios 2(8): 138-150.

Del Moral-Flores L, J Tello-Musi, H Reyes-Bonilla, H Pérez-España, J Martínez-Pérez, G Horta-Puga, L Velazco-Mendoza \& P Álvarez del Castillo-Cárdenas. 2013. Lista sistemática y afinidades zoogeográficas de la ictiofauna del Sistema Arrecifal Veracruzano, México. Revista Mexicana de Biodiversidad 84(3): 825-846.

Doherty PJ, V Dufour, R Galzin, MA Hixon, MG Meekan \& S Planes. 2004. High mortality during settlement is a population bottleneck for a tropical surgeonfish. Ecology 85(9): 2422-2428.

Dower JF, TJ Miller \& WC Leggett. 1997. The role of microscale turbulence in the feeding ecology of larval fish. Advances in Marine Biology 31: 170-220.

Espinosa-Fuentes ML, C Flores-Coto, F Zavala-García, L Sanvicente-Añorve \& R Funes-Rodríguez. 2013. Seasonal vertical distribution of fish larvae in the southern Gulf of Mexico. Hidrobiológica 23(1): 42-59.

Fahay MP. 2007. Early stages of fishes in the Western North Atlantic Ocean (Davis Strait, Southern Greenland and Flemish Cap to Cape Hatteras). Journal North West Atlantic Fishery Science I-II: 1-1681.

Flores-Coto C, ML Espinosa-Fuentes, F Zavala-García \& L Sanvicente-Añorve. 2009. Ictioplancton del sur del Golfo de México. Un compendio. Hidrobiológica 19(1): 49-76.

Froese R \& D Pauly. 2014. FishBase. <http://fishbase.org>

García-Hernández V, U Ordóñez-López, T HernándezVázquez \& JN Álvarez-Cadena. 2009. Fish larvae and juveniles checklist (Pisces) from the North of the Yucatan Peninsula, Mexico with 39 new records for the region. Instituto de Biología, Universidad Nacional Autónoma de México. Revista Mexicana de Biodiversidad 80: 85-94.

Grigg RW, JJ Polovina \& MJ Atkinson. 1984. Model of a coral reef ecosystem III. Resource limitation, community regulation, fisheries yield and resource management. Coral Reefs 3: 23-27.

Jiménez-Badillo ML \& LG Castro-Gaspar. 2007. Pesca artesanal en el Parque Nacional Sistema Arrecifal Veracruzano, México. En: Granados-Barba A, LG AbarcaArenas \& JM Vargas-Hernández (eds). Investigaciones científicas en el Sistema Arrecifal Veracruzano, pp. 221-240, Universidad Autónoma de Campeche, Campeche.

Jiménez-Valverde A \& J Hortal. 2003. Las curvas de acumulación de especies y la necesidad de evaluar la calidad de los inventarios biológicos. Revista Ibérica de Aracnología 8: $151-161$.

Leis JM. 1993. Larval fish assemblages near Indo-Pacific coral reefs. Bulletin of Marine Science 53(2): 362-392.

Leis JM. 2006. Are larvae of demersal fishes plankton or nekton? Advances in Marine Biology 51: 59-141. 
Leis JM \& MI McCormick. 2002. The biology, behavior, and ecology of the pelagic larval stage of coral reef fishes. In: Sale PF (ed). Coral reef fishes: Dynamics and diversity in a complex ecosystem, pp. 171-199. Academic Press, San Diego.

Martínez-Hernández JA \& B Camacho-Olivares. 2007. Ictiofauna del arrecife artificial 'Excañonero C-50, General Vicente Riva Palacio' en el Sistema Arrecifal Veracruzano, México. En: Granados-Barba A, LG Abarca-Arenas \& JM Vargas-Hernández (eds). Investigaciones Científicas en el Sistema Arrecifal Veracruzano, pp. 209-220. Universidad Autónoma de Campeche, Campeche.

Okolodkov YB, JA Aké-Castillo, MG Gutiérrez-Quevedo, H Pérez-España \& D Salas-Monreal. 2011. Annual cycle of the plankton biomass in the National Park Sistema Arrecifal Veracruzano, Soutwestern Gulf of Mexico. In: Kattel G (ed). Zooplankton and phytoplankton, pp. 63-88. Nova Science Publishers, Hauppauge.

Ordóñez-López U, R Huchin-Sel, J Peniche-Pérez, S Gallegos-Fernández, R Sosa-Pinto \& L VásquezYeomans. 2013. Variación temporal del ictioplancton colectado con trampas de luz en los puertos de Chuburná y Yucalpetén, Yucatán, México. Hidrobiológica 23(2): 265-273.

Ortiz-Lozano LD, A Granados-Barba \& I Espejel. 2009. Ecosystemic zonification as a management tool for marine protected areas in the coastal zone: Applications for the Sistema Arrecifal Veracruzano National Park, Mexico. Ocean and Coastal Management 52: 317-323.

Ortiz-Lozano L, A Granados-Barba, I Espejel, J SalasPérez \& C González-Gándara. 2015. La zona costera de Veracruz a Antón Lizardo: un análisis sobre la vulnerabilidad de sus servicios ambientales. E-Bios 2(8): 151-178.

Poot-Delgado CA, YB Okolodkov, JA Aké-Castillo \& J Rendón-von Osten. 2015. Annual cycle of phytoplankton with emphasis on potentially harmful species in oyster beds of Términos Lagoon, southeastern Gulf of Mexico. Revista de Biología Marina y Oceanografía 50(3): 465-477.

Rangel-Avalos MA, LKB Jordan, BK Walker, DS Gilliam, E Carvajal-Hinojosa \& R Spieler. 2007. Fish and coral reef communities of the Parque Nacional Sistema Arrecifal Veracruzano (Veracruz Coral Reef System National Park) Veracruz, México: Preliminary results. Proceedings of the 60th Gulf and Caribbean Fisheries Institute, pp. 427-435. Punta Cana, Rep. Dominicana.

Reséndez-Medina A. 1971. Peces colectados en el arrecife La Blanquilla, Veracruz, México. Anales del Instituto de Biología, Universidad Nacional Autónoma de México, Serie Ciencias del Mar y Limnología 42: 7-30.

Richards WJ. 2006. Early stages of Atlantic fishes. An identification guide for the Western Central North Atlantic 1 \& 2: 1-2640. CRC Taylor \& Francis, New York.

Salas-Monreal D, DA Salas-de-León, MA Monreal-Gómez \& ML Riverón-Enzástiga. 2009. Current Rectification in a Tropical Coral Reef System. Coral Reefs 28(4): 871-879.
Salas-Pérez J \& A Granados-Barba. 2008. Oceanographic characterization of the Veracruz Reefs System. Atmósfera 21(3): 281-301.

Salas-Pérez JJ, D Salas-Monreal, MA Monreal-Gómez, ML Riveron-Enzastiga \& C Llasat. 2012. Seasonal absolute acoustic intensity, atmospheric forcing and currents in a tropical coral reef system. Estuarine Coastal and Shelf Science 100: 102-112.

Sale PF. 2004. Connectivity, recruitment variation, and the structure of reef fish communities. Integrative and Comparative Biology 44: 390-399.

Sánchez-Velasco L, C Flores-Coto \& B Shirasago. 1996. Fish larvae abundance and distribution in the coastal zone off Terminos Lagoon, Campeche (Southern Gulf of Mexico). Estuarine Coastal and Shelf Science 43: 707-721.

Sanvicente-Añorve L. 1990. Comunidades ictioplanctónicas en el suroeste del Golfo de México. Tesis de Maestría, Facultad de Ciencias, Universidad Nacional Autónoma de México, $60 \mathrm{pp}$.

Sanvicente-Añorve L, M Sánchez-Ramíres, A Ocaña-Luna, C Flores-Coto \& U Ordóñez-López. 2011. Metacommunity structure of estuarine fish larvae: the role of regional and local processes. Journal of Plankton Research 33(1): 179-194.

Smith PE \& SL Richardson. 1977. Standard techniques for pelagic fish and larvae surveys. FAO Fisheries Technical Paper 175: 1-99.

Soberón J \& J Llorente. 1993. The use of species accumulation functions for the prediction of species richness. Conservation Biology 7: 480-488.

Urho L. 1999. Relationship between dispersal of larvae and nursery areas in the Baltic Sea. ICES Journal of Marine Science 56: 114-121.

Vargas-Hernández JM, A Hernández-Gutiérrez \& LF Carrera-Parra. 1993. Sistema Arrecifal Veracruzano. En: Salazar-Vallejo S \& NE González (eds). Biodiversidad marina y Costera de México, pp. 559-575. CONABIO-CIQRO, México.

Vargas-Hernández JM, G Nava-Martínez \& MA RománVives. 2002. Peces del Sistema Arrecifal Veracruzano. En: Guzmán-Anaya P, C Quiroga-Brahms, C Díaz-Luna, D Fuentes-Castellanos, CM Contreras \& G Silva-López (eds). La pesca en Veracruz y sus perspectivas de desarrollo, pp. 17-29. Universidad Veracruzana y Centro Regional de Investigación Pesquera, México.

Villalobos-Figueroa A. 1971. Estudios ecológicos en un arrecife coralino en Veracruz, México, En: Coloquio sobre investigaciones y recursos del mar Caribe y regiones adyacentes, pp. 531-545. UNESCO, Paris.

Villegas-Sánchez CA, H Pérez-España, R Rivera-Madrid, D Salas-Monreal \& JE Arias-González. 2014. Subtle genetic connectivity between Mexican Caribbean and southwestern Gulf of Mexico reefs: the case of the bicolor damselfish, Stegastes partitus. Coral Reefs 33: 241-251. 